\section{Phaselocked tunable subcarrier comb generator}

S.-L. Tsao, J. Wu, T.-M. Chen and J.S. Wu

Indexing terms: Optical communication, Phaselocked loops, Phase modulation

A novel tunable subcarrier comb generator has been realised by dual-in-line phase modulators with optimum phase modulation dual-in-line phase modulators with optimum phase modulation
indices. An optical heterodyne and microwave homodyne indices. An optical heterodyne and microwave homodyne
phaselocked loop is used to lock all optical carriers simultaneously. The hold-in range and linewidth are $\sim 20 \mathrm{MHz}$ and $20 \mathrm{~Hz}$, respectively.

Introduction: Subcarrier multiplexing (SCM) and frequency division multiplexing (FDM) schemes present an attractive approach to the design of broad-band optical communication systems [1, 2] Subcarrier or optical comb generators (OCGs) have the potential to provide extremely dense channels in SCM and FDM optical communication systems, and the application of phase array microwave antenna systems [3]. Obviously, flattened, dense and phase stabilised subcarriers or optical channel carriers are essential for realising these schemes. Various methods to achieve comb generator have been proposed [5].

In this Letter, we demonstrate a method to achieve phaselocked tunable subcarrier comb generators (TSCGs) with two cascade phase modulators by optical phaselocked loop (OPLL) technology. The OPLL technology also provides a convenient method fo microwave homodyne and optical heterodyne phaselocking of optical carriers, and can be used to synchronise the laser frequencies in multioptical carrier communication systems. To determine the optimum phase modulation indices to achieve a maximally flattened optical comb spectrum, the theoretically relative power standard deviations of the optical carriers of the OCG are calculated. The experimental performance of the OPLL is also reported.

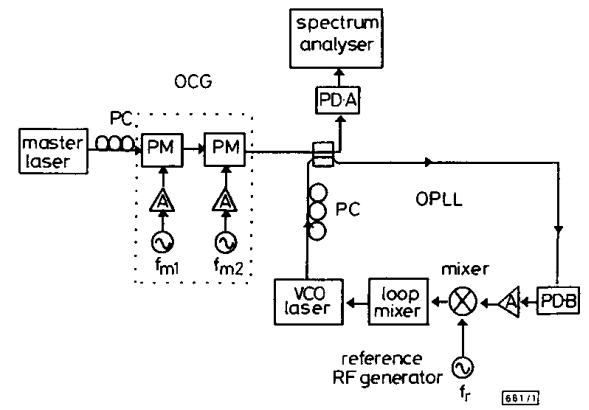

Fig. 1 Schematic diagram of experimental setup of phaselocked TSCG

PM: phase modulator, PD: pin photodetector, PC: polarisation controller, OPLL: optical phaselocked loop, OCG: optical comb generato

Principle of TSCG: The block diagram of the experimental setup of the phaselocked TSCG is shown in Fig. 1. Two tunable diodepumped Nd:YAG lasers operating around $1.3 \mu \mathrm{m}$ are used as the master and VCO lasers. The light from the master laser is master and VCO lasers. The light from the master laser is
launched into the dual-in-line phase modulators, which are driven launched into the dual-in-line phase modulators, which are driven by two high power microwave tones $f_{1}$ and $f_{2}$. Thus, with large
phase modulation indices $\beta_{1}$ and $\beta_{2}$, we can obtain a large number phase modulation indices $\beta_{1}$ and $\beta_{2}$, we can obtain a large number optical field of the comb carriers can be written as

$E_{\text {comb }}=\sqrt{P_{m}} \cos \left\{2 \pi f_{m} t+\beta_{1} \sin \left(2 \pi f_{1} t\right)+\beta_{2} \sin \left(2 \pi f_{2} t\right)\right]$

where $P_{m}$ and $f_{m}$ are the power and frequency of the master laser. To obtain equal channel spacing, $f_{2}$ should be a harmonic frequency of $f_{1}$. Therefore, it is possible to change the channel spacing by choosing the correct $f_{1}, f_{2}, \beta_{1}$ and $\beta_{2}$ to obtain a relatively flat optical comb spectrum. The optical carriers from the OCG are mixed with the VCO laser output by a $2 \times 2$ optical coupler, while the optical field of the VCO laser can be represented as

$$
E_{V C O}=\sqrt{P_{v}} \cos \left(2 \pi f_{v} t\right)
$$

where $P_{v}$ and $f_{v}$ are the power and frequency of the VCO laser.

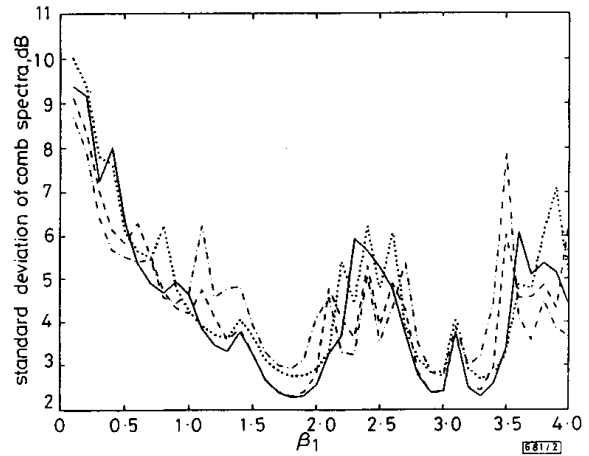

Fig. 2 Standard optical power deviation of comb carriers for different $\boldsymbol{\beta}_{2}$ against $\boldsymbol{\beta}$

$$
\begin{aligned}
& \ldots \cdots \cdots \beta_{2}=2.7 \\
& \cdots \cdots \beta_{2}=2.9 \\
& \cdots-\cdot \beta_{2}=3.1 \\
& \cdots-\beta_{2}=3.3
\end{aligned}
$$

The heterodyned comb spectra detected by the photodiode PD A are monitored by a microwave spectrum analyser. The output $\mathrm{AC}$ of the photodiode can be expressed as

$$
\begin{aligned}
i^{A C}(t) & \propto R \sqrt{P_{m} P_{v}} \\
& \times \sum_{n=-\infty}^{\infty} \sum_{k=-\infty}^{\infty} J_{n}\left(\beta_{1}\right) J_{k}\left(\beta_{2}\right) \cos 2 \pi\left(f_{I F}+n f_{1}+k f_{2}\right) t
\end{aligned}
$$

where $R$ denotes the responsivity of the photodiode, $J_{n}\left(\beta_{1}\right)$ and $J_{k}\left(\beta_{2}\right)$ represent the $n$th and $k$ th order Bessel functions of the first kind and $f_{I F}\left(=\left|f_{m}-f_{v}\right|\right)$ is the intermediate frequency. To implement the phaselocked TSCG, we use an OPLL to lock the phase of the selected carrier as shown in Fig. 1. The VCO laser of the OPLL tracks the phase and the frequency of the selected carrier using a phase stabilising feedback loop. In this heterodyne OPLL, the OCG and VCO lasers oscillate at different frequencies to produce a set of microwave carriers after photodiode PD B. A mixer duce a set of microwave carriers after photodiode PD B. A mixer
functioning as an RF phase detector compares the phases of the selected carrier and the reference signal $f_{r}$ and generates the phase error signal which is further processed by an RC filter $(\tau=5 \mathrm{~ms})$ and then applied to the PZT of the VCO laser to form the OPLL. Using 24 carriers to perform numerical optimisation, we obtain the standard deviation of carrier power of the OCG for various $\beta_{2}$ as a function of $\beta_{1}$, shown in Fig. 2 . From the numerical results shown in Fig. 2, we find that some local optimum values as $\beta_{1}=$ $1.8, \beta_{2}=2.9 ; \beta_{1}=3.3, \beta_{2}=2.9 ; \beta_{1}=2.9, \beta_{2}=2.9 ; \beta_{1}=3.0, \beta_{2}=$ 3.1 etc. can provide relatively flat comb spectra.

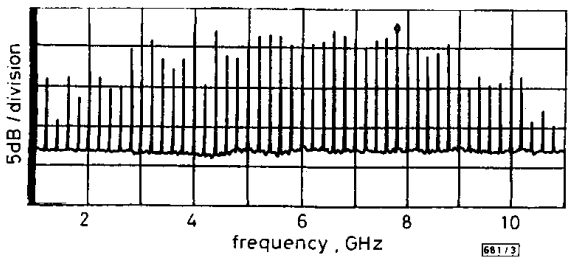

Fig. 3 Comb spectrum measured at $f_{1}=200 \mathrm{MHz}, f_{2}=1.2 \mathrm{GHz}, f_{I F}=$ Marker: $7.8 \mathrm{GHz},-22.35 \mathrm{dBm}$ 
Experimental results: We choose the same phase modulation index $\beta \approx 2.9$ for the two phase modulators. The RF modulation frequencies for the dual-in-line phase modulators are set at $f_{1}=$ $200 \mathrm{MHz}$ and $f_{2}=1.2 \mathrm{GHz}$, respectively. Fig. 3 shows the measured phaselocked optical comb carriers with reference RF frequency $f_{r}=2 \mathrm{GHz}$. We can choose the centre region of 24 carriers which gives the maximum power deviation below $5 \mathrm{~dB}$ as subcarriers for applications. An expanded view of the optical comb spectra ers $\mathrm{Fi}$ s shows the linewich of a selected optical carier in Fig 4. With a minimum resolution bandwidth of $10 \mathrm{~Hz}$ (which is limited by the RF spectrum analyser), we can see that the $3 \mathrm{~dB}$ linewidth of each carrier is below $20 \mathrm{~Hz}$

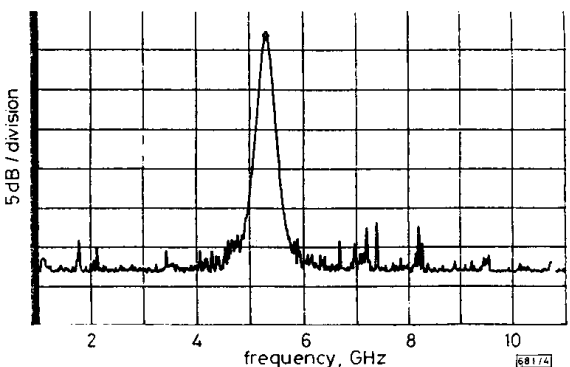

Fig. 4 Spectrum of one carrier of comb carriers under phaselocked condition

Vertical: $5 \mathrm{~dB}$ /division, horizontal: centre: $1.999998800 \mathrm{GHz}$, Span:

Marker: $1.999998731 \mathrm{GHz},-23.18 \mathrm{dBm}$

The VCO laser is operating at $1319 \mathrm{~nm}$ with $1 \mathrm{~mW}$ optical output power. A PZT element mounted on the Nd:YAG crystal provides fast frequency tuning. The frequency, tuning coefficient of vides fast frequency tuning. The frequency, tuning coefficient of the PZT is $5.5 \mathrm{MHz} / \mathrm{V}$. We find that this OPLL can provide a
hold-in range of $20 \mathrm{MHz}$. When the desired lockiug RF frequency falls into the range of $400 \mathrm{kHz}$ away from reference frequency $f$ the pull-in process of the OPLL drives the VCO laser slowly unti the beat frequency approaches the reference frequency. Therefore, the pull-in range is $400 \mathrm{kHz}$ in our experiment. Furthermore the lock-in range we measured is $\sim 48 \mathrm{kHz}$ over which the OPLL acquires phaselocking without cycle slips.

Conclusions: We have implemented a tunable phaselocked optica comb generator by mixing two laser lights with one light bein appropriately phase modulated. With the optimal modulation indices $\beta_{1}=\beta 2.9$ of the phase modulators, a phaselocked 24 carindices comb $\beta_{1}$ PLL tracking technique with a hold-in range of $20 \mathrm{MHz}$ is employed to achieve phaselocking for all the carriers generated by the OCG simultaneously. The $3 \mathrm{~dB}$ linewidth of each optical carrier is less than $20 \mathrm{~Hz}$. The pull-in range and lock-in range are $400 \mathrm{kHz}$ and $48 \mathrm{kHz}$, respectively. This scheme shows the potential of using lasers to generate tunable phaselocked narrow linewidth optical comb carriers for SCM and FDM optical com munication systems or optical-controlled microwave phase array antennas.

(C) IEE 1994

Electronics Letter, Online No: 19941377

16 September 1994

S.-L. Tsao, J. Wu, T.-M. Chen and J.-S. Wu (Department of Electrical Engineering. National Taiwan University. Taipei. Taiwan, Republic of China)

\section{References}

1 GROSS. R., and OLSHANSKY, R.: 'Multichannel coherent FSK experiments using subcarrier multiplexing techniques', J. Lightwave Technol., 1990, LT-8, pp. 406-415

2 NOSL. K., TOBA, H., INOUE, K, and ODA, K: '100 channel optical FDM technology and its applications to optical FDM channelbased networks', J. Lightwave Technol,, 1993, LT-11, pp. 764-776

3 SURETTE. M.R., HJELME, D.R., and MICKELSON, A.R.: "An optically driven phased array antenna utilizing heterodyne technique.. $J$. Lightwave Technol., 1993, LT-11, pp. 1500-1509
4 HODGKINSON. T.G., and COPPIN. P.: 'Comparison of sinusoidal and pulse modulation techniques for generating optical frequency

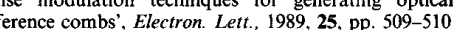

5 WU, J.S., TSAO. S.L., TSAO. H.-W., and WU, J.: 'Tunable subcarrier comb generator using phase modulator with high modulation index. SPIE, 1993, 2024, pp. 286-294

\section{Satellite coupler: Application to distributed} star network

T. Ota

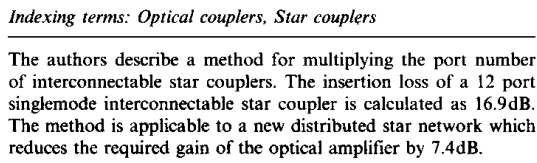

Introduction: Recently, there have been some reports on networks constructed by directly connecting star couplers. Networks constructed with conventional star couplers using sophisticated connection methods were reported in $[1,2]$. Networks using interconnectable star couplers and simple connection methods were reported in [3-8]. The direct connection of star couplers involving optical amplifiers, was reported in $[2,5,7,8]$. Star couplers having more terminals are preferred for many applications.

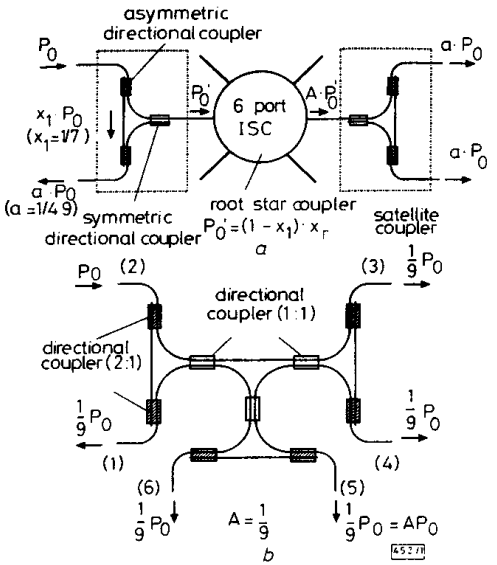

Fig. 1 Method to multiply port number of interconnectable star coupler 6 port $\rightarrow 12$ port $)$

$a$ Connection of satellite coupler

Structure of 6 port interconnectable star coupler

Multiplication method: Fig. $1 a$ shows a method for doubling the port number of a 6 port singlemode interconnectable star couple (ISC). Satellite couplers are connected to the root interconnectable star coupler. The satellite coupler consists of two asymmetric directional couplers and one symmetric directional coupler as shown in Fig. 1a. The internal structure of a 6 port interconnectable star coupler [7] is shown in Fig. $1 b$.

As described in a previous paper [7], the distribution ratio of an asymmetric directional coupler $x_{1}$ is derived as follows:

$$
\begin{aligned}
& a=x_{1}^{2} \\
& a=\left(1-x_{1}\right)^{2} x_{r}^{2}-t
\end{aligned}
$$

where $a, x_{r}$ and $A$ are the insertion loss (transmission coefficient) where $a, x_{r}$ and $A$ are the insertion loss (transmission coefficient)
of a 12 port interconnectable star coupler, distribution ratio of a 